\title{
Chinese immigrant smokers' access barriers to tobacco cessation services and experience using social media and text messaging
}

\author{
Nan Jiang', Yidan Zhang', Xiaokun Qian', Lorna Thorpe', Chau Trinh-Shevrin', Donna Shelley²
}

\begin{abstract}
INTRODUCTION Smoking rates remain disproportionately high among Chinese immigrants in the US, particularly in males. Community-based smoking cessation services and quitlines have low engagement rates. Social media and text messaging programs can be effective in promoting quit rates and improving treatment engagement. This study examined Chinese immigrant smokers' barriers to accessing available smoking cessation services and patterns of using social media platforms and mobile phone text messaging.

METHODS We conducted in-depth interviews $(n=30)$ and a brief survey $(n=49)$ with adult Chinese immigrant smokers leaving in New York City in 2018. Qualitative interviews explored smokers' challenges with smoking cessation, barriers to accessing and using smoking cessation services, and experience using social media and text messaging. The quantitative survey assessed smoking and quitting behaviors, and social media and text messaging use patterns.

RESULTS Qualitative data revealed that participants faced various barriers to accessing cessation services, including the lack of awareness about services, skepticism about treatment effects, reliance on willpower for cessation, and time constraints. WeChat was mainly used to maintain social networking and acquire information. Participants rarely used text messaging or other social media platforms. Quantitative data showed that $55 \%$ of participants had no plan to quit smoking. Among those who reported past-year quit attempts (45\%), 55\% used cessation assistance. WeChat was the most frequently used platform with 94\% users.

CONCLUSIONS WeChat has potential to serve as an easily accessible platform for delivering smoking cessation treatment among Chinese immigrant populations. Research is warranted to explore the feasibility and efficacy of employing WeChat in smoking cessation treatment.
\end{abstract}

\section{AFFILIATION}

1 Department of Population Health, Grossman School of Medicine, New York University, New York, United States

2 School of Global Public Health, New York University, New York, United States

CORRESPONDENCE TO Nan Jiang. Department of Population Health, Grossman School of Medicine, New York University, 180 Madison Ave, New York, NY 10016, United States. E-mail: Nan.Jiang2@nyulangone. org ORCID ID: https://orcid. org/0000-0003-0714-750X

\section{KEYWORDS}

mobile health, smoking, cessation, Chinese immigrant

Received: 29 May 2020

Revised: 18 July 2020

Accepted: 2 August 2020

\section{INTRODUCTION}

Smoking rates remain disproportionately high among Chinese immigrants, particularly in males. In New York City (NYG), the city with the largest Chinese population in the $\mathrm{US}^{1,2}$, the current (past 30-day) smoking rate among Chinese American males is significantly higher than that among the general male population $(28.2 \% \text { vs } 17.5 \%)^{3}$. Foreign-born Chinese Americans (representing $72 \%$ of Chinese American population in NYG) ${ }^{4}$ are more likely to smoke than their US-born counterparts ${ }^{3}$.

The persistent high smoking rate among Chinese immigrants is in part due to the low intention to quit and underutilization of evidence-based smoking cessation services such as telephone quitlines and in-person counseling programs ${ }^{5,6}$. Studies among Chinese American smokers reported that 35-51\% were not interested in quitting, and most of those 
who tried to quit relied on willpower without using any proven smoking cessation methods ${ }^{5,7-9}$. The low quit intention may be attributed to low health literacy about the harms of smoking ${ }^{5,9,10}$ and strong attachment to traditional Chinese social norms that support smoking in men ${ }^{11,12}$. The underuse of proven cessation methods may be explained by the dearth of culturally appropriate programs targeting this population ${ }^{13}$ and access barriers to available smoking cessation services ${ }^{14-16}$. Current smoking cessation programs, which focus on Chinese communities such as Asian Smokers' Quitline (ASQ), and communitybased services have limited population impact due to their low engagement rates ${ }^{17}$.

Addressing tobacco-related disparities for Chinese immigrants and maximizing the impact of tobacco control among this population requires more understanding about their challenges in using available cessation services. It also requires innovative forms of interventions that can increase treatment reach and effectiveness. Mobile health (mHealth) strategies, such as automated text messaging or short message service (SMS) programs and social media-based (e.g. Facebook or Twitter) smoking cessation interventions are effective in promoting quit outcomes ${ }^{18-21}$, and can reach wide audiences and deliver time-sensitive support. Metaanalyses on SMS smoking cessation interventions reported that the interventions increased 7-day point prevalence abstinence (odds ratio, $\mathrm{OR}=1.38$; 95\% CI: $1.22-1.55)^{21}$ and 26-week abstinence rates (risk ratio, $\left.\mathrm{RR}=1.67 ; 95 \% \mathrm{CI}: 1.46-1.90 ; \mathrm{I}^{2}=59 \%\right)^{20}$. A systematic review of social media-based smoking cessation interventions (i.e. via Facebook, Twitter and WhatsApp) concluded that the interventions had promising preliminary effectiveness in increasing quit outcomes ${ }^{19}$. Therefore, SMS and social media cessation interventions may expand smokers' access to smoking cessation treatment.

However, Chinese immigrants may primarily use WeChat, a multi-purpose social media app that is popular in China. Launched in 2011 by Tencent, WeChat has reached 1.2 billion monthly active users worldwide as of May 2020, ranking the 3rd most popular mobile messenger app worldwide after WhatsApp and Facebook Messenger ${ }^{22}$. Roughly $83 \%$ of smartphone users in China use WeChat ${ }^{23}$. Despite the widespread use in China, WeChat use pattern among Chinese immigrant populations in US remains unknown. The purpose of this study was to explore Chinese immigrant smokers' quitting experiences, and their social media and mobile phone text messaging usage. Findings from this study may help inform strategies to improve engagement and effectiveness of smoking cessation interventions that focus on this racial/ ethnic minority group that has high smoking rates and disproportionately lacks access to cessation treatment.

\section{METHODS}

From March through May 2018, we employed indepth interviews among 30 adult Chinese immigrant smokers in NYC. Using a semi-structured guide, we conducted in-depth interviews to explore their smoking cessation experiences, barriers to accessing and using available smoking cessation services, and experience using social media and mobile phone text messaging. Eligibility criteria for the in-depth interview included: 1 ) aged $\geq 18$ years, 2) Chinese immigrants, 3) current smokers or former smokers who quit in the past 12 months, and 4) used WeChat in the past 6 months. We requested participants to have experience with WeChat because it would allow them to provide insights about what they like and dislike about WeChat, use patterns and more. Interviews lasted 30-55 minutes and were conducted in Mandarin, the official language of China. Interviews were audio-taped, transcribed verbatim, and translated into English.

Following the in-depth interviews, we developed a quantitative survey to assess Chinese immigrant smokers' smoking patterns, quit attempts and intention, past-year use of cessation aids, and patterns of using social media and mobile phone text messaging. Eligible participants for the quantitative survey were Chinese immigrant current smokers aged $\geq 18$ years, and they were not required to have ever used WeChat. A total 49 Chinese immigrant smokers completed the self-administered paper-andpencil survey in May and June 2018. Two of the 49 participants completed both the qualitative interview and quantitative survey.

Social media and text messaging use was assessed by asking 'On average, how often do you use WeChat?' and 'On average, how often do you do 
the following things on WeChat: instant messaging to individuals; instant messaging in groups; read news/articles; post on the "Moments"; browse friend/family's posts; play games; transfer money or make payment?'. Answer options ranged from 'several times a day' to 'never'. Participants rated the frequency of using WeChat, WhatsApp, Facebook, Twitter, Instagram, and mobile phone text messaging on a 1-7 visual analog scale (' 1 ' labeled as 'least frequently', ' 7 ' as 'most frequently'), or chose 'I don't use it'.

Participants were recruited via flyer postings and in-person contacts (snowballing) from Chinatown (Manhattan) and Flushing (Queens), two communities with high concentrations of Chinese immigrant populations in NYC. Participants received $\$ 50$ for the qualitative interview and $\$ 15$ for survey. Study protocols were approved by New York University Grossman School of Medicine's Institutional Review Board.

Qualitative data were analyzed using NVivo $12^{24}$. An initial codebook of themes and subthemes related to research questions was created using inductive analytical techniques of qualitative data analyse ${ }^{25,26}$.
Three team members reviewed the codebook, discussed the appropriateness of the themes and subthemes, coded a subset of randomly selected transcripts independently, revised the codebook by adding emergent themes and subthemes, resolved disagreements on the codes through iterative discussions to reach consensus and theme saturation. Two team members then independently coded all of the transcripts. Descriptive statistics summarized quantitative survey results (e.g. frequency and percentage) using Stata $14.2^{27}$.

\section{RESULTS \\ Qualitative findings}

Participants (aged 19-61 years) included 6 females and 24 males, 3 former smokers and 27 current smokers. Four primary themes emerged, including low quit intention, challenges to smoking cessation, access barriers to available smoking cessation services, and prevalent WeChat use (Table 1).

\section{Low quit intention}

Participants were generally not interested in quitting smoking. While smokers were familiar with the

\section{Table 1. In-depth interview themes and illustrative quotations}

\section{Theme 1: Low quit} intention

\section{Low awareness} about the harms of smoking

Misconceptions about smoking cessation

To relieve stress

To maintain social connections

Denial of being addicted
'Cigarette is not like drugs such as heroin that causes tremendous harms to health... If you smoke for a decade or two, or even thirty or forty years, you will get lung cancer. But it doesn't make other damages to your body. Smoking may make you skin age faster, but that's a slow process. Nobody cares...I don't plan to quit because there are no immediate harms of smoking.' (P2, male)

'I don't think smoking affects my health or life. So I have never thought about quitting.' (P5, male)

'My family doctor told me that my lungs are a bit dark. But all smokers' lungs are a little dark...Smoking doesn't seem to cause any obvious health issues to me. I don't really suffer from lung cancer or any other diseases... I quit before, and did not smoke for 6 months. I felt nothing changed before and after I quit. It seems there is no difference in my health conditions. So forget about quitting. I smoked again.' (P7, male)

'Many people say that quitting smoking is harmful and you won't benefit anything from quitting. Mao Zedong suffered from health issues after he quit smoking. So did Deng Xiaoping. These great men both experienced health problems after they quit smoking.' (P8, male)

'I feel anxious when I don't do well. Smoking helps relieve my anxiety. I know smoking is not good for health. But I depend on it mentally. There is no other way [to relieve anxiety].' (P17, male)

'Smoking for me is a way to reduce pressure...I'm not interested in gambling or drinking alcohol to reduce pressure.' (P21, male)

'Smoking itself doesn't attract me. But it's like a bridge, helping me know friends better and improve our relationship... Some of my coworkers smoke. I offer them cigarettes and they also offer me cigarettes. We smoke together. That makes it easier for us to get along...' (P22, male) 'When I smoke with friends, I really feel that I can better fit in with my friends.' (P25, male)

'I haven't tried to quit because I don't smoke a lot. I am not addicted. So it doesn't matter whether to quit or not.' (P9, male) 
Table 1. Continued

Themes

Quotations

\section{Theme 2:}

Challenges to

smoking cessation

Cigarette offering 'My coworkers smoked and they offered me cigarettes. So I relapsed.' (P10, male)

custom

Lack of craving

management skills

Theme 3: Access

barriers to

available smoking

cessation services

Unfamiliarity with cessation services

Skepticism about treatment efficacy

Tendency of relying on willpower

Time constraints

Misconceptions about cessation services

Theme 4: Prevalent WeChat use

Use experience

Attitudes toward WeChat

Concerns about WeChat (P18, male) why I'm not aware of those services.' (P8, male) quit.' (P15, male) to quit, I personally think that the success rate won't be high.' (P22, male) work. We don't return home until 10pm or even later'. (P18, male) rarely use it.' (P2, male) WeChat [than Facebook/Twitter].' (P3, male) for years. For Instagram, I am not interested. I like WeChat.' (P4, female) male)
'After smoking for years, I am addicted to cigarettes. When I try to quit, I feel loss of energy and saliva is tasteless. It's very uncomfortable. I couldn't taste anything. This is the biggest challenge for me.' (P23, male) 'The biggest challenge for me is that when I am bored or in bad mood or feel stressful, I really want to smoke.'

'I am not aware of smoking cessation clinics or quitlines. I have been here [in NYC] for 4 years only. Maybe that's

'I think the counseling services only provide very basic information. That does not work.' (P27, male)

'Willpower is the key [to smoking cessation]. Nicotine patches or other medicines or whatever won't help people

'Smoking cessation services play a supporting role. The willpower is more important. If you rely on those services

'People like us don't have time to visit cessation clinics or call a quitline. We get up at 8am or 9am, and then go to

'Those services [cessation clinics and quitlines] are for heavy smokers. I guess only very heavy smokers need those services...I don't need those services, because my case is not that serious.' (P28, female)

'I use mobile phone text messaging but very rarely. I only text if the contact person is a non-acquaintance or if I have no other contact information...I seldom use Facebook, maybe once or twice a month, because my friends

'I don't use Facebook or Twitter...Most people in my social circle are Chinese. It's more convenient for me to use

'I seldom use mobile phone text messaging. I only text foreigners [non-Chinese], because only foreigners use text messages. All my other contacts are on WeChat...I have Facebook and Twitter accounts, but I haven't used them

'Before, we contacted friends by phone calls. Now we use WeChat. Voice messages or video calls are so convenient. They are faster [than text messages], easy and convenient. I feel like WeChat brings people closer'. (P3,

'WeChat makes life easier and more convenient. It makes it easier for me to connect with friends, family and relatives. I also get a lot of information and learn new things through WeChat. It's so convenient. I personally think WeChat is great. There is nothing I don't like about it. I like WeChat 100 percent.' (P14, male)

'I definitely have concerns about WeChat. I use it a lot and all my personal information is there, including my phone number and chat history. If someone wants to check on me, they can get all my information on WeChat.' (P17, male)

'I worry that Tencent would release user's information.' (P6, female) generic health warning that smoking is harmful, most were uncertain about the exact harms of smoking and benefits of quitting. A common misconception was that quitting smoking would endanger smokers' health rather than reduce their risks, particularly for those who have smoked for years. One person said: 'Mao Zedong suffered from health issues after he quit smoking, so did Deng Xiaoping. These great 
men both had health problems after quitting' (P8, male). Furthermore, smoking was regarded by many participants as the only way to cope with bad mood (e.g. homesick and loneliness) and stress. As firstgeneration immigrants, participants experienced a significant decline in the size of social circles because of immigration, and they generally worked long hours. These could increase their risks for depression and anxiety. But they did not know how to deal with bad mood and stress without smoking. Participants said: 'I live on my own here in NYC. I smoke when I get homesick or have nothing to do' (P15, male) and 'I feel anxious when I don't do well. Smoking helps relieve my anxiety' (P17, male). Male smokers described offering cigarettes to friends as an important social activity to establish and maintain social connections. One participant said: 'For Chinese, we sometimes need to offer cigarettes to others in order to build relationships. So I have to smoke' (P11, male). Finally, non-daily smokers tended to deny that they were addicted, which made them consider cessation as unnecessary.

\section{Challenges to smoking cessation}

The persistent social norms, such as offering cigarettes to friends and not feeling comfortable refusing offered cigarettes posed a major challenge for quitting. One smoker said: 'All people around me smoke. I told them that I've quit. They said "Smoking one more cigarette is fine". So it's impossible for me to quit' (P4, female). The other challenge is the lack of strategies to cope with cravings, as a smoker said: 'When I saw other people smoking, I could not hold it. Quitting made me feel sleepy so I relapsed' (P26, male).

Access barriers to available smoking cessation services Most participants did not know that smoking cessation clinics and quitlines such as ASQ were available, and had no information about treatment procedures or how to use the services. One person noted: 'I've never heard about cessation clinics or quitlines. There is no information about their treatment procedures' (P2, male). Participants expressed skepticism about treatment efficacy, as one smoker said: 'You can quit by chatting with someone? I don't think it's gonna happen' (P1, male). In addition, smokers perceived willpower as key to successful quitting and consider cessation treatment as noncomparable to a strong willpower. One person said: 'My willpower can help me quit. I'd prefer not to use things like smoking cessation clinics or quitlines' (P12, female). Moreover, participants' busy life hampered them from attending smoking cessation clinics or ASQ. Smokers said: 'It's burdensome to use cessation clinics and quitlines. You have to make phone calls, make appointments, and visit doctors' (P26, male) and 'People like us don't have time to visit cessation clinics or call quitlines. We get up at 8 am or 9 am, and then go to work. We don't return home until $10 \mathrm{pm}$ or even later' (P18, male). Finally, non-daily smokers thought that cessation services were designed for heavy smokers. Thus, they did not even consider using the services.

\section{Prevalent WeChat use}

Nearly all participants used WeChat on a daily basis. One smoker said: 'Everyone uses WeChat. I feel like there is no way to keep a normal social life if you don't use WeChat' (P17, male). Participants reported that WeChat voice messaging and voice/video call made communication 'easier and more convenient' (P3, male), and WeChat 'Moments' allowed them to easily 'get updates about families and friends' (P5, male) and 'share personal joyful and torturous experiences with friends' (P14, male). Participants also enjoyed reading articles and news through WeChat. One participant said: 'Now I seldom read newspapers. I read news on WeChat every day' (P24, male).

Participants generally had no concerns about WeChat, because the privacy setting allowed users to define who can view their 'Moments'. One smoker said: 'I think most Chinese have no concerns. Otherwise they would not post things like where they go, where they eat, or share their locations on the Moments' (P4, female). Five participants expressed concerns like 'I worry that Tencent would release users' information' (P6, female).

Participants used mobile phone text messaging but on rare occasions. Few participants used other social media platforms. According to the users, text messaging was used only to contact non-Chinese or non-acquaintance, Facebook and Instagram to keep connections with non-Chinese friends, and WhatsApp to contact people living in Hong Kong. Participants compared WeChat and Facebook, and felt 'more secure to use WeChat because it protects users' privacy' (P6, female). One smoker noted: 'On 
Facebook, everyone can see your posts. Whereas on WeChat, only confirmed friends can see your posts' (P26, male).

\section{Quantitative findings}

Participants' mean age was 47.9 years $(\mathrm{SD}=13.27)$ (Table 2). Most were males (80\%) and were born in mainland China (94\%). About 31\% of participants completed primary or middle school, $43 \%$ completed high school, $8 \%$ received associate degree from college, and $18 \%$ received Bachelor's or advanced degrees. Participants had lived in the US for an average of 10.3 years $(\mathrm{SD}=9.22)$. About $73 \%$ were married, and $63 \%$ were full-time employed.

Most participants $(86 \%)$ were daily smokers. Participants' mean age of smoking initiation was 19.9 years $(\mathrm{SD}=8.25)$. On average, participants smoked 13.8 cigarettes per day ( $\mathrm{SD}=8.49)$. Among those who reported past-year quit attempts (45\%), 55\% used smoking cessation methods including $14 \%$ visiting a smoking cessation clinic. No one had called a quitline. Over half of respondents (55\%) had no plan to quit smoking. Onethird of participants had ever used e-cigarettes.

The majority $(n=46 ; 94 \%)$ of participants used WeChat, including 90\% daily users $(n=44)$. WeChat was primarily used for social networking ( $80 \%$ daily browsing the 'Moments', $76 \%$ and $50 \%$ daily sending messages to individuals and in groups respectively) and information acquisition (67\% daily reading

Table 2. Sociodemographic characteristics and tobacco use among Chinese immigrant smokers $(\mathrm{N}=49)$

$\begin{array}{lcr}\text { Characteristics } & n & (\%) \\ \text { Age (years), mean (SD) } & 47.9 & (13.27) \\ \text { Gender } & 39 & (79.59) \\ \text { Male } & 10 & (20.41) \\ \text { Female } & & \\ \text { Place of birth } & 46 & (93.88) \\ \text { Mainland China } & 3 & (6.12) \\ \text { Other } & 10.3 & (9.22) \\ \text { Residence in the US (years), mean (SD) } & & \\ \text { Education } & 15 & (30.61) \\ \text { Middle school or less } & 21 & (42.86) \\ \text { High school or vocational high school } & 4 & (8.16) \\ \text { Some college, no degree or associate degree } & 9 & (18.37) \\ \text { Bachelor's or advanced degree } & & \text { Continul }\end{array}$

Table 2. Continued

$\begin{array}{lrr}\text { Characteristics } & n & (\%) \\ \text { Marital status } & & \\ \text { Married, living with spouse } & 32 & (65.31) \\ \text { Married, living apart with spouse } & 4 & (8.16) \\ \text { Never married or single } & 9 & (18.37) \\ \text { Divorced or widowed } & 4 & (8.16)\end{array}$

Working status

Full-time employed 31

Part-time employed

Other

Age of smoking initiation (years), mean (SD) $19.9 \quad$ (8.25)

Current smoking status

Non-daily smoker

Daily smoker 42

Cigarettes per day, mean (SD)

Time to first cigarette after waking (minutes)

$<5$

$6-30$

$31-60$

$>60$

Don't know

Quit attempt in the past 12 months

No

Yes

Quit methods $(\mathrm{n}=22)$

Gave up cigarettes all at once

Cut down on cigarettes gradually

Visited smoking cessation clinics

Consulted other healthcare professionals

Used nicotine replacement therapy

Called a quitline

Read self-help materials

Read information through the internet

Used an e-cigarette

Quit intention

Have no plan to quit 27

Plan to quit within the next 6 months

Plan to quit within the next 30 days

Trying to quit now

Ever use of alternative tobacco products ${ }^{\mathrm{b}}$

E-cigarette

Waterpipe

Cigar

Chewing tobacco, snuff or dip

Snus or dissolvable tobacco product

a Among current smokers who tried to quit in the past 12 months $(n=22)$. Multiple responses, do not add up to $100 \%$. b Multiple responses, do not add up to $100 \%$. 
Figure 1. Frequency of using social media platforms and mobile phone text messaging

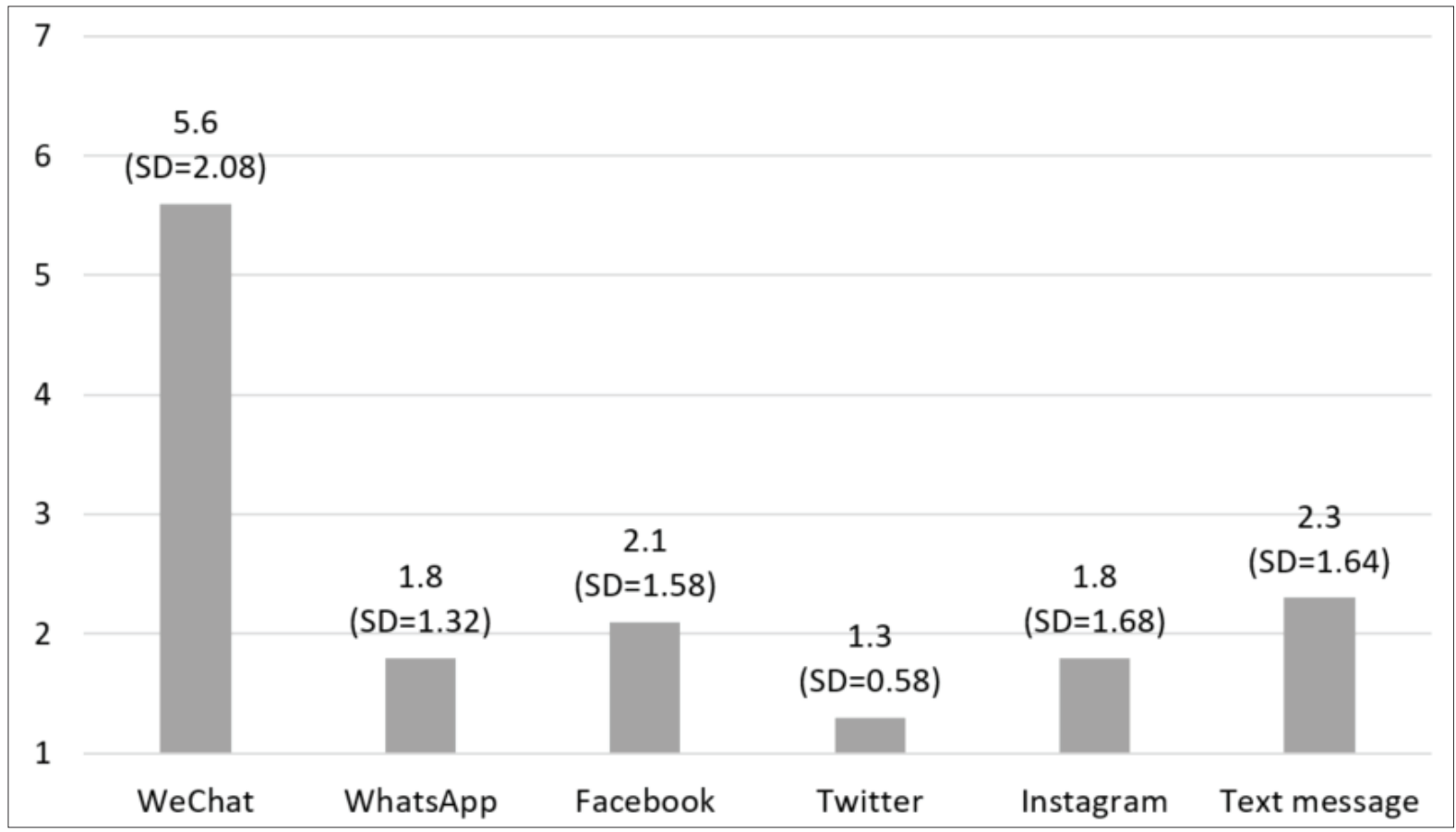

Participants rated their frequency of using each platform on a 1-7 visual analog scale, with '1' labeled as 'least frequently' and '7' as 'most frequently'. Those who checked 'I don't use it' were excluded. SD: standard deviation.

news/articles; data not shown). Few participants used Facebook $(\mathrm{n}=11 ; 22 \%)$, WhatsApp $(\mathrm{n}=10$; $20 \%)$, Instagram $(n=5 ; 10 \%)$, or Twitter $(n=3 ; 6 \%)$. All but one used mobile phone text messaging. WeChat was the most frequently used platform (5.6, $\mathrm{SD}=2.08$ ) followed by mobile phone text messaging $(2.3, \mathrm{SD}=1.64)$ and Facebook $(2.1, \mathrm{SD}=1.58)$ (Figure 1).

\section{DISCUSSION}

This is among the few studies that investigated challenges in smoking cessation and barriers to accessing available smoking cessation services among Chinese immigrants, a disadvantaged group with high smoking rates and a lack of access to evidencebased cessation treatment. It is also among the first efforts to assess social media and text messaging use in this population. Our study affirmed the low quit intention among Chinese immigrant smokers ${ }^{5,8,9}$ and provided insights into why they were not interested in quitting. Smokers were not entirely clear about the risks of smoking. Consistent with prior studies ${ }^{11,15}$, the misconception that quitting smoking would lead to health problems is common. Although the Centers for Disease Control and Prevention has invested in large scale anti-smoking media campaigns, most have not targeted Chinese Americans ${ }^{28}$. This may explain their low awareness about the harms of smoking. Future interventions should not assume this knowledge among this population and need to include information about how exactly smoking harms the body and what specific benefits people would gain from quitting.

Consistent with prior research ${ }^{11,12,14,15}$, our findings highlight the prominent role of customs that support offering cigarettes to friends and norms that create discomfort in refusing, as a major barrier to quitting. Male smokers practiced this custom to maintain social networks. Encouragingly, participants acknowledged that this custom is less pervasive in the US, indicating that smokers may be sensitive to the American anti-tobacco societal values. Future cessation programs targeting Chinese immigrant smokers need to provide skill training in refusal strategies. Messages emphasizing considerations for others' health and cessation decision may help reframe the cigarette offering culture.

The lack of stress management skills also 
explained Chinese immigrant smokers' low quit intention. As first-generation immigrants, smokers often worked long hours and had limited social support due to language barriers and the decline in social circle size. These factors posed elevated risks for depression and anxiety. Without stress coping capacities, many chose to ignore the adverse health effects and continue to smoke. Smoking cessation programs targeting this population should provide training in stress management.

Smokers, including men and women, encountered similar access barriers to available smoking cessation services, including their low awareness about the services, doubt of treatment effects, misconception about the effect of willpower, and time constraints. Educational campaigns that inform people about the proven cessation methods, their treatment procedures and efficacy, and encourage smokers to try those methods as a complement to willpower may promote the uptake of proven cessation methods. Moreover, Chinese immigrant smokers may be reluctant to use counseling therapy (e.g. smoking cessation clinics and quitlines) for making behavioral changes ${ }^{16}$. Studies are warranted to explore innovative forms of smoking cessation treatment that promotes engagement among this population.

Non-daily smokers' perceptions about addiction played an important role in their cessation decision making. They tended to deny addiction and believed that cessation services are designed for heavy smokers. Thus, they did not even think about quitting or seeking cessation assistance. Findings suggest that Chinese immigrant smokers may not fully understand nicotine dependence. Future research is warranted to understand how Chinese smokers conceptualize addiction. Smoking cessation programs need to include discussions about risks and symptoms of addiction.

As an important social networking tool and primary source of information and entertainment, WeChat was the most frequently used social media platform among Chinese immigrant smokers. Other platforms and mobile phone text messaging were rarely used. WeChat's prompt and convenient communication features allowed people to maintain close interactions with families and friends. The recreational feature (e.g. reading news/articles) caught people's attention whenever they felt bored or craved for distractions. Most participants had no concern about WeChat. Few expressed their concern about Tencent's capacity to protect user information. Such concern is common for most social media platforms. Given the prevalent use, WeChat has potential to serve as an easily accessible platform for delivering smoking cessation treatment among Chinese immigrant smokers. To our knowledge, no study has been conducted in the US to explore using WeChat in tobacco control outreach or treatment programs. A few studies have been carried out in China to evaluate WeChat-based smoking cessation treatment, but efficacy remains unknown ${ }^{29}$. Future research is necessary to investigate the feasibility and effectiveness of using WeChat to engage Chinese immigrant smokers and deliver smoking cessation treatment.

\section{Limitations}

Findings should be interpreted with caution. First, our sample was relatively small, and participants were recruited from two communities in NYC. Thus, we cannot assume the findings to be broadly representative. Second, qualitative interviews were conducted in Mandarin. Thus, beliefs and experiences of Cantonese-speaking smokers and those who speak other dialects were not captured. However, the majority of Chinese immigrants in NYC can speak Mandarin even if their primary language is Cantonese $^{30}$. Third, our study was conducted before the COVID-19 crisis. Smokers may have changed their smoking behaviors since the outbreak of the pandemic because coronavirus poses for smokers an increased risk of serious pulmonary complications compared to non-smokers. A recent meta-analysis reviewed 19 studies and concluded that smoking was associated with higher odds of progression in COVID-1931.

\section{CONCLUSIONS}

This study has important implications for future research. Given the prevalent WeChat use, research is warranted to explore the feasibility and efficacy of using WeChat for smoking cessation treatment targeting Chinese immigrant populations. Moreover, treatment programs for Chinese immigrant smokers must address their low awareness about risks of smoking, correct misconceptions about cessation and willpower, reframe social norms around smoking and 
offer training in stress and craving management skills and refusal strategies.

\section{REFERENCES}

1. New York City Department of City Planning. Population - New York City population: Population facts. https:// www1.nyc.gov/site/planning/data-maps/nyc-population/ population-facts.page. Accessed May 4, 2018.

2. United States Census Bureau. Selected population profile in the United States: 2016 American community survey 1-year estimates. https://centropr.hunter.cuny.edu/ sites/default/files/data_sheets/ACS_16_1YR_S0201\%20 US.pdf. Published September 14, 2017. Accessed May 4, 2018.

3. New York City Department of Health and Mental Hygiene. Epi Data Brief https://www1.nyc.gov/assets/ doh/downloads/pdf/epi/databrief103.pdf. Published June, 2018. Accessed March 2, 2019.

4. Haines-Saah RJ, Kelly MT, Oliffe JL, Bottorff JL. Picture Me Smokefree: a qualitative study using social media and digital photography to engage young adults in tobacco reduction and cessation. J Med Internet Res. 2015;17(1):e27. doi:10.2196/jmir.4061

5. Shelley D, Fahs M, Scheinmann R, Swain S, Qu J, Burton D. Acculturation and tobacco use among Chinese Americans. Am J Public Health. 2004;94(2):300-307. doi:10.2105/ajph.94.2.300

6. Tsang IK, Tsoh JY, Wong C, et al. Understanding and use of nicotine replacement therapy and nonpharmacologic smoking cessation strategies among Chinese and Vietnamese smokers and their families. Prev Chronic Dis. 2014;11:E26. doi:10.5888/pcd11.130299

7. Ma GX, Tan Y, Toubbeh J, Su X. Differences in stages of change of smoking behavior among current smokers of four Asian American subgroups. Addict Behav. 2003;28(8):14311439. doi:10.1016/s0306-4603(03)00071-6

8. Fu SS, Ma GX, Tu XM, Siu PT, Metlay JP. Cigarette smoking among Chinese Americans and the influence of linguistic acculturation. Nicotine Tob Res. 2003;5(6):803811. doi:10.1080/14622200310001614566

9. Spigner C, Yip M-P, Huang B, Shigaki A, Tu SP. Chinese and Vietnamese adult male smokers' perspectives regarding facilitators of tobacco cessation behavior. Asian Pac J Cancer Prev. 2007;8(3):429-435. PMID:18159982.

10. Yu ESH, Chen EH, Kim KK, Abdulrahim S. Smoking among Chinese Americans: Behavior, knowledge, and beliefs. Am J Public Health. 2002;92(6):1007-1013. doi:10.2105/ajph.92.6.1007

11. Zhang JY, Chan SSC, Fong DYT, Malone RE, Lam TH. The social context of smoking cessation in China: an exploratory interview study. Tob Control. 2012;21(1):5758. doi:10.1136/tc.2010.040691

12. Spence W, Zhu L. Perceptions of smoking cessation among Glasgow's Chinese community. Tobacco Prevention \&
Cessation. 2017;3(October):1-9. doi:10.18332/tpc/77942

13. Chen MS, Tang H. Review of smoking cessation research among Asian Americans: The state of the research. Nicotine Tob Res. 2007;9(3):S485-S493. doi:10.1080/14622200701587052

14. Hsia FN, Spruijt-Metz D. The meanings of smoking among Chinese American and Taiwanese American college students. Nicotine Tob Res. 2003;5(6):837-849. doi:10.1080/14622200310001615259

15. Tu SP, Walsh M, Tseng B, Thompson B. Tobacco use by Chinese American men: An exploratory study of the factors associated with cigarette use and smoking cessation. Asian Am Pac Isl J Health. 2000;8(1):46-57. PMID:11567512.

16. Katigbak C, Maglalang DD, Chao YY, Au H, Liang W, Zuo S. Cultural perspectives on tobacco use and cessation among Chinese American immigrants: A community-engaged qualitative study. J Transcult Nurs. 2019;30(4):350-358. doi:10.1177/1043659618817583

17. Kuiper N, Zhang L, Lee J, et al. A national Asian-language smokers' quitline - United States, 2012-2014. Prev Chronic Dis. 2015;12:1-10. doi:10.5888/pcd12.140584

18. Kong G, Ells DM, Camenga DR, Krishnan-Sarin S. Text messaging-based smoking cessation intervention: A narrative review. Addict Behav. 2014;39(5):907-917. doi:10.1016/j.addbeh.2013.11.024

19. Naslund JA, Kim SJ, Aschbrenner KA, et al. Systematic review of social media interventions for smoking cessation. Addict Behav. 2017;73:81-93. doi:10.1016/j.addbeh.2017.05.002

20. Whittaker R, McRobbie H, Bullen C, Rodgers A, Gu Y. Mobile phone-based interventions for smoking cessation. Cochrane Database Syst Rev. 2016;4(4):CD006611. doi:10.1002/14651858.CD006611.pub4

21. Scott-Sheldon LA, Lantini R, Jennings EG, et al. Text messaging-based interventions for smoking cessation: A systematic review and meta-analysis. JMIR Mhealth Uhealth. 2016;4(2):e49. doi:10.2196/mhealth.5436

22. Statista. Most popular global mobile messenger apps as of July 2019, based on number of monthly active users (in millions). https://www.statista.com/statistics/258749/ most-popular-global-mobile-messenger-apps/. Published July, 2019. Updated July, 2020. Accessed November 19, 2019.

23. Hollander R. WeChat has hit 1 billion monthly active users. Business Insider. https://www.businessinsider. com/wechat-has-hit-1-billion-monthly-activeusers-2018-3. Published March 6, 2018. Accessed July $18,2020$.

24. QSR International Pty Ltd. NVivo qualitative data analysis software. Version 12. Doncaster, Australia: QSR International Pty Ltd; 2018. https://www. qsrinternational.com/nvivo-qualitative-data-analysissoftware/home. Accessed July 18, 2020.

25. Lewins A, Silver C. Using Software in Qualitative 
Research: A Step-by-Step Guide. London, UK: SAGE Publications; 2007.

26. Saldaña J. The Coding Manual for Qualitative Researchers. 1st ed. London, UK: SAGE Publications; 2009. https:// iribresearch.ir/rm/book/The $\% 20$ coding $\% 20$ manual $\% 20$ for $\% 20$ qualitative $\% 20$ researchers $\% 20$ - Johnny_ Salda\%C3\%B1a-2009.pdf. Accessed July 18, 2020.

27. StataCorp. Stata Statistical Software. Version 16. College Station, TX: StataCorp; 2019.

28. Centers for Disease Control and Prevention. Tips From Former Smokers ${ }^{\circledR}$ : About the campaign. https://www. cdc.gov/tobacco/campaign/tips/about/index.html?s_ cid=OSH_tips_D9393. Published April 23, 2018. Accessed June 16, 2018.

29. Li WHC, Ho KY, Lam KKW, et al. A study protocol for a randomised controlled trial evaluating the use of information communication technology (WhatsApp/ WeChat) to deliver brief motivational interviewing (i-BMI) in promoting smoking cessation among smokers with chronic diseases. BMC Public Health. 2019;19(1):1083. doi:10.1186/s12889-019-7417-6

30. Semple K. In Chinatown, sound of the future is Mandarin. The New York Times. https://www. nytimes.com/2009/10/22/nyregion/22chinese. html?pagewanted=1\&em. Published October 21, 2009. Accessed July 18, 2020.

31. Patanavanich R, Glantz SA. Smoking is associated with COVID-19 progression: A meta-analysis. Nicotine Tob Res. 2020;22(9):1653-1656. doi:10.1093/ntr/ntaa082

CONFLICTS OF INTEREST

The authors have completed and submitted the ICMJE Form for Disclosure of Potential Conflicts of Interest and none was reported.

\section{FUNDING}

This study was supported by the New York University Center for the Study of Asian American Health under the National Institute on Minority Health and Health Disparities (NIMHD) of the National Institutes of Health (U54MD000538-15). NIMHD played no role in study design, data collection and analysis, manuscript writing, or the decision to submit the paper for publication.

PROVENANCE AND PEER REVIEW

Not commissioned; externally peer reviewed. 